\title{
Magnetostatic interactions in a natural magnetite-ulvöspinel system
}

\author{
Michael E. Evans, ${ }^{1}$ David Krása, ${ }^{2}$ Wyn Williams, ${ }^{2}$ and Michael Winklhofer ${ }^{3}$ \\ Received 18 April 2006; revised 6 July 2006; accepted 25 July 2006; published 20 October 2006.
}

[1] Magnetostatic interactions have been investigated in an intergrown material consisting of $\sim 200$-nm magnetite blocks separated by $\sim 30$-nm-wide ulvöspinel lamellae.

First-order reversal curve (FORC) measurements provide a direct measure of the interaction fields, giving a value for the full width at half maximum (FWHM) of $30 \mathrm{mT}$ (at $H_{\mathrm{c}}=20 \mathrm{mT}$ ). Hysteresis parameters are $M_{r s} / M_{s}=0.22, H_{c r} / H_{c}=1.98$ and transient energy dissipation $(\mathrm{TED})=0.18$. Elimination of the intergrowth structure (by heating in vacuo) causes the FORC contours to shrink down toward the origin, yielding FWHM $=14 \mathrm{mT}$ (at $H_{c}=6 \mathrm{mT}$ ) with corresponding changes in $M_{r s} / M_{s}, H_{c r} / H_{c}$ and TED to $0.11,2.73$ and 0.28 , respectively. All these characteristics reveal the strong influence of particle-to-particle magnetostatic interactions between the magnetite blocks in the starting material and demonstrate the change from single-domain/pseudosingle-domain to multidomain behavior due to the fundamental structural change brought about by the experimental homogenization. Micromagnetic calculations of particle assemblages representative for the intergrown structure confirm that the magnetite blocks will interact by assuming a supervortex magnetization structure.

Citation: Evans, M. E., D. Krása, W. Williams, and M. Winklhofer (2006), Magnetostatic interactions in a natural magnetiteulvöspinel system, J. Geophys. Res., 111, B12S16, doi:10.1029/2006JB004454.

\section{Introduction}

[2] It is well known that magnetostatic interactions strongly influence the hysteresis properties of natural and artificial samples, but their effects are difficult to quantify. This would be something of an academic question were it not for the fact that situations in which interactions are likely to be important are by no means rare. In the laboratory, for example, experiments with magnetic extracts are universally plagued by the problem of suitably dispersing the mineral grains in order to obtain the elusive goal of an ideal noninteracting configuration. In rocks, the important paleomagnetic minerals (mostly iron-titanium oxides) often occur in the form of microscopic intergrowths in which neighboring magnetic regions lie in close proximity to one another. It is this latter problem with which we are primarily concerned here. A particularly striking example has recently been described by Feinberg et al. [2005].

[3] In an early attempt to monitor the effect of ultrafine subdivision of mineral phases Evans and Wayman [1974] measured the remanence properties of a rock containing intergrown magnetite/ulvöspinel grains. The main result of relevance to paleomagnetism was that the intergrown grains have much higher coercivities than their experimentally homogenized counterparts. This, in turn, implies greater time stability for the single-domain/pseudosingle-domain

\footnotetext{
${ }^{1}$ Department of Physics, University of Alberta, Edmonton, Alberta, Canada.

${ }^{2}$ School of GeoSciences, University of Edinburgh, Edinburgh, UK.

${ }^{3}$ Department of Earth and Environmental Sciences, Ludwig-Maximilians-Universität, Munich, Germany.

Copyright 2006 by the American Geophysical Union. 0148-0227/06/2006JB004454\$09.00
}

(SD/PSD) intergrowths compared to the multidomain (MD) homogenized material. The inevitability of magnetostatic interactions between the tiny magnetite regions was recognized, but no attempt was made to consider them quantitatively. Recent developments, on three broad fronts, have made a renewed effort very much worthwhile. First, vastly increased computing power now makes it possible to calculate the behavior of arrays of magnetic particles that can be regarded as model intergrown systems [Muxworthy et al., 2004; Muxworthy and Williams, 2005]. Second, the evolution of the Preisach model [Preisach, 1935] into the so-called first-order reversal curve (FORC) analysis [Pike et al., 1999; Roberts et al., 2000], with its attendant instrumental automation, now provides a direct experimental measure of interaction fields. Third, electron holography is now able to provide detailed images of the magnetic field pattern inside samples of interest. Indeed, it was the remarkable images obtained by Harrison et al. [2002] of the same material originally studied by Evans and Wayman [1974] that prompted the present enquiry.

\section{Experimental Procedure}

[4] Hysteresis and thermomagnetic measurements were made on a variable field translation balance (VFTB) in the Munich laboratory. For this purpose, we packed into a tubular quartz holder a $2.8 \mathrm{mg}$ aliquot of the same mineral separate used in the original study of Evans and Wayman [1974]. This takes the form of a powder with a typical grain size of about $20 \mu \mathrm{m}$, but with a distribution extending beyond $100 \mu \mathrm{m}$. The subdivision of the grains by the intergrowth structure produces roughly rectangular magnetite blocks with typical dimensions about 3 orders of 


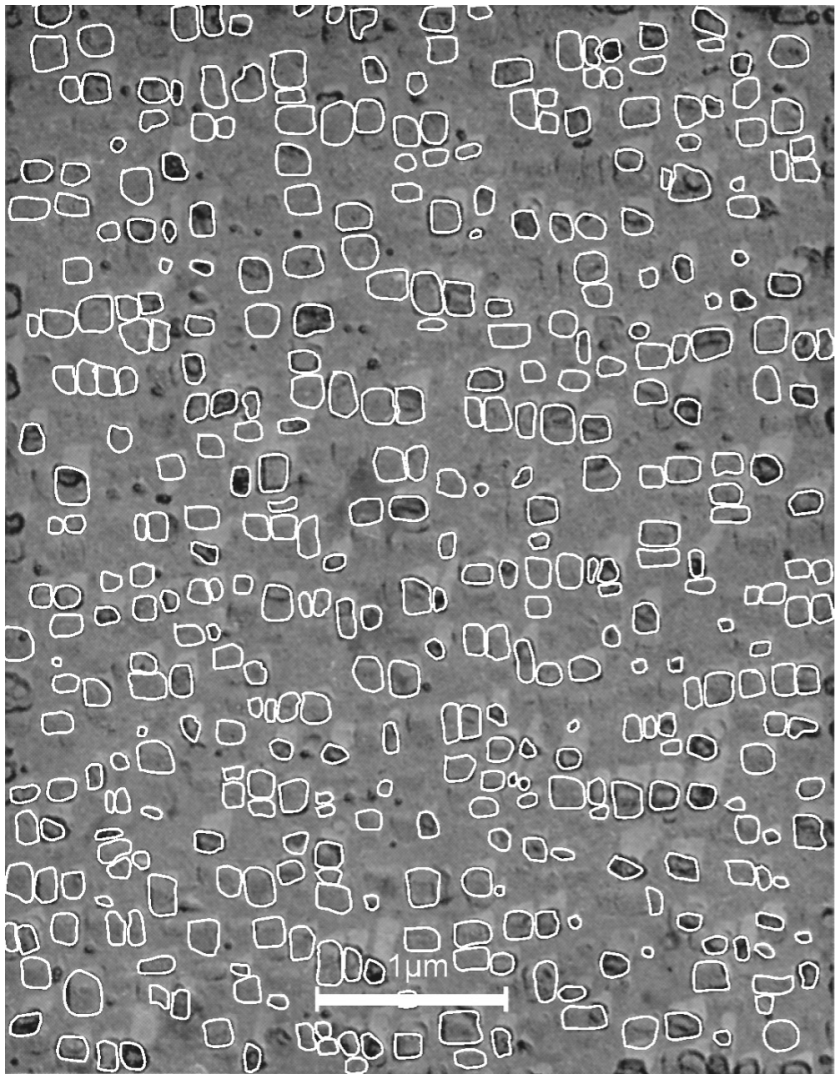

Figure 1. Replica technique transmission electron micrograph of an intergrown grain from the Yamaska Mountain ore body, Québec. The replica consists of a carbon film preshadowed with a gold-palladium alloy. Outlines of the magnetite blocks used in our size analysis are indicated. (Original image obtained by Evans and Wayman [1974].)

magnitude smaller. To better characterize the size and shape distributions of the magnetite subregions we used an automated size-analyzing algorithm (Laboratory Imaging Ltd., LUCIA 4.6). The perimeters of 407 magnetite blocks were digitized manually as shown in the TEM image reproduced in Figure 1. The software then determined the minimum and maximum Feret's diameter of each block. From that, the long and short axes of the approximately rectangular magnetite blocks were calculated to yield the particle elongation. First-order reversal curves (FORC) were determined at the University of Utrecht Palaeomagnetic Laboratory using a Princeton Measurements Corporation MicroMag instrument with custom in-house software.

[5] In order to assess the effect of the intergrowth structure and its concomitant drastic reduction in effective grain size, we repeated the FORC and VFTB hysteresis measurements on samples that had been experimentally homogenized by the procedure of Evans and Wayman [1974]. An 8-mg aliquot was sealed under vacuum into a small quartz vial, held at $1000^{\circ} \mathrm{C}$ for 2 hours and then quenched by rapidly immersing the vial in water.

\section{Results}

[6] The thermomagnetic curve obtained from the VFTB measurements is shown in Figure 2. A very clear Curie point of $548^{\circ} \mathrm{C}$ is obtained by the extrapolation method of Moskowitz [1981], indicating that the rectangular blocks seen in the TEM images are not quite pure magnetite. The well-established relationship between composition and Curie point implies that their actual composition is $\mathrm{Fe}_{2.94} \mathrm{Ti}_{0.06} \mathrm{O}_{4}$, in good agreement with the final endmember composition expected from the phase diagram [Basta, 1960].

[7] Figure 3 shows the VFTB hysteresis loop obtained (Figure 3a) as well as the isothermal remanent magnetization (IRM) acquisition and backfield curves (Figures $3 \mathrm{~b}$ and 3c). The magnetization and coercivity ratios $M_{r s} / M_{s}$ and $H_{c r} / H_{c}$ are 0.22 and 1.98 , respectively. The transient energy dissipation (TED) proposed by Fabian [2003] was found to be 0.18 . The $H_{c r} / H_{c}$ value is close to that expected near the $\mathrm{SD} / \mathrm{PSD}$ boundary, but the $M_{r s} / M_{s}$ value is much lower than the expected theoretical value for noninteracting SD particles and the TED value is significantly higher. We attribute these deviations from ideal SD behavior to interactions among the particles, although an alternative possibility is that particles slightly above the SD/PSD threshold have vortex state remanence configurations with reduced $M_{r s} / M_{s}$ ratios. We favor interactions as the main factor, since this is consistent with the FORC diagrams (see below) and with the electron holography images of Harrison et al. [2002]. Harrison et al.'s Figure 3 clearly demonstrates that strong interaction between neighboring magnetite blocks leads to what they refer to as magnetic superstates, with much reduced, even zero, net magnetization. This is also consistent with the size data we derived from the TEM image shown above. The measured grain sizes agree with data determined by Evans and Wayman [1974] and are summarized in Figure 4 where we plot the maximum dimension of each measured block against its axial ratio (Figure 4a) as well as the distribution of maximum dimensions and the gaps between nearest neighbors (Figure 4b). According to the theoretical calculations of Fabian et al. [1996], it appears that many of the blocks are, indeed, too large to behave as true SD particles. However, it is also clear, as is obvious from a glance at Figure 1, that the magnetite blocks are almost always closer together than their typical dimensions.

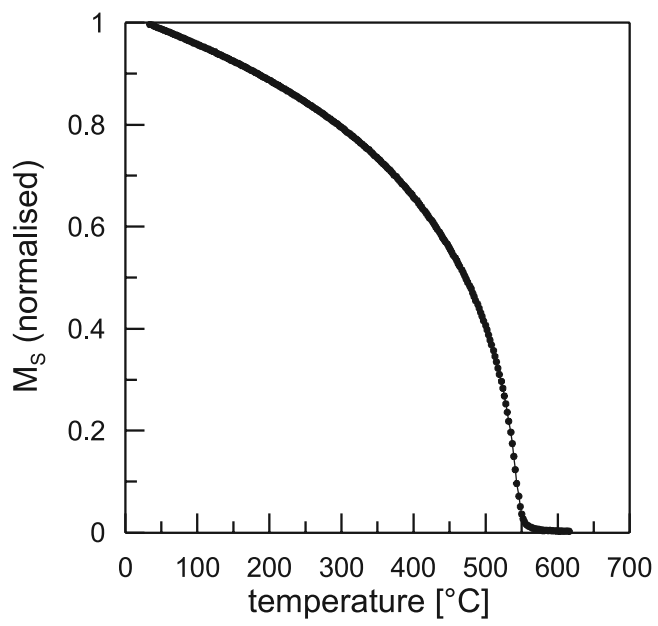

Figure 2. Thermomagnetic curve for the intergrown material. A Curie point of $548^{\circ} \mathrm{C}$ is obtained from extrapolation method of Moskowitz [1981]. 

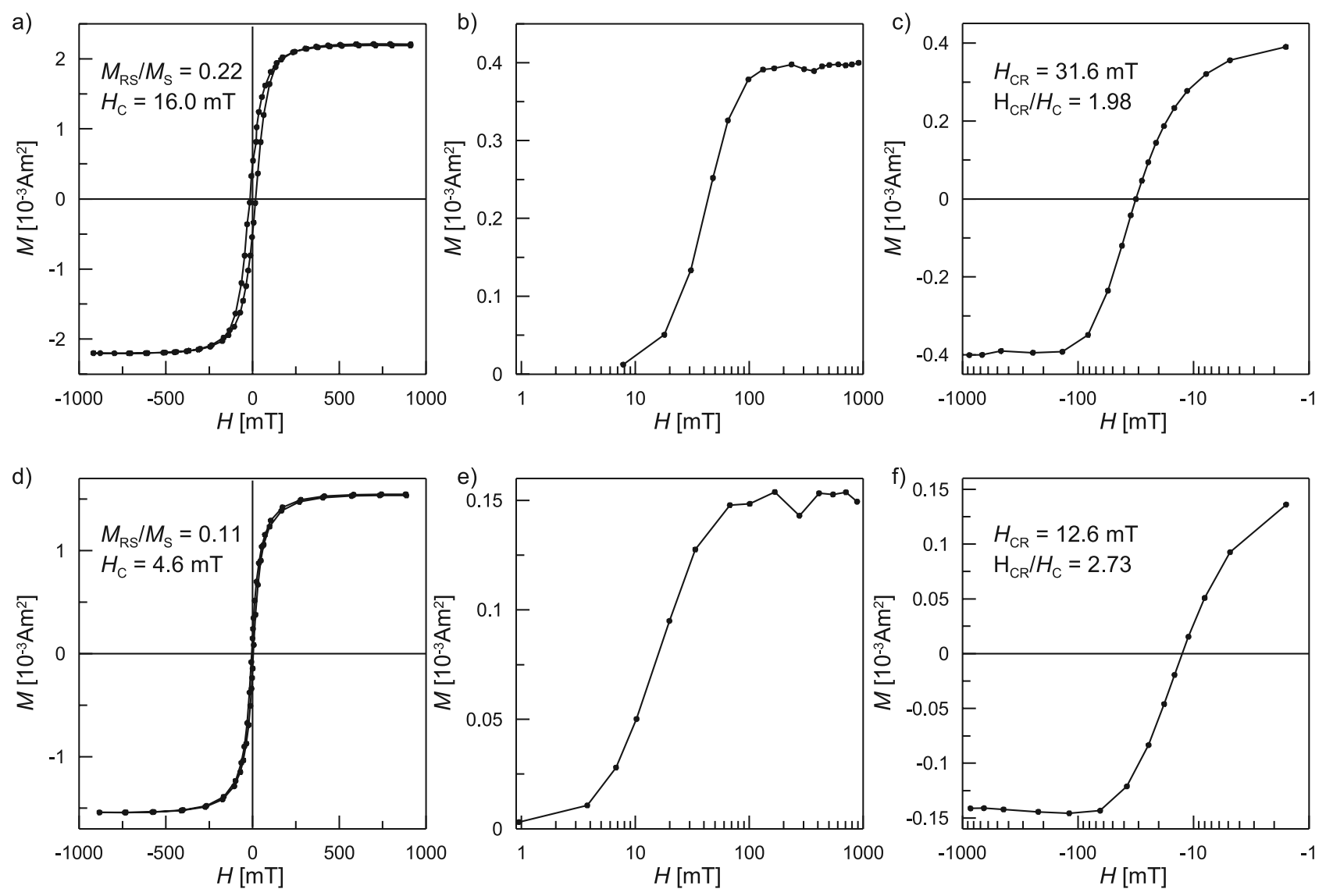

Figure 3. (a) Hysteresis loop, (b) IRM acquisition curve and (c) backfield curve for the intergrown material; (d) hysteresis loop, (e) IRM acquisition curve and (f) backfield curve for the homogenized material.

The maximum dimensions follow a normal distribution with a median value of $195 \mathrm{~nm}$, whereas the gaps between nearest neighbors are exponentially distributed with a median value of $27 \mathrm{~nm}$ (in both cases, $R>0.99$ ). In the terminology of
Muxworthy et al. [2004], $d \ll 1$. Magnetostatic interactions must therefore be of paramount importance.

[8] The FORC diagram for the intergrown material is given in Figure 5a. In this kind of plot the underlying
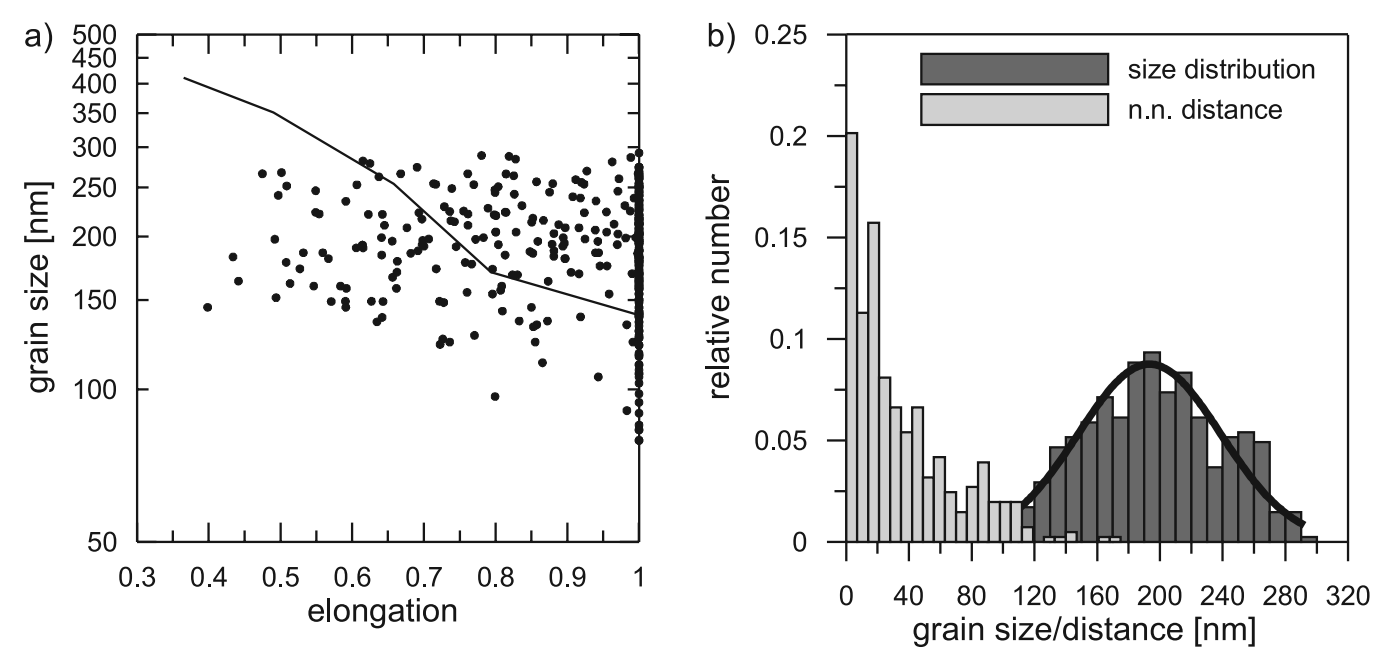

Figure 4. (a) Size and shape distribution of magnetite blocks. The black line corresponds to the SDPSD threshold derived by Fabian et al. [1996]. (b) Distributions of maximum dimensions and gaps between nearest neighbors (n.n.). Since Figure 1 represents a random section (i.e., it is not necessarily a (100) plane), all these dimensions are maxima. Black curve is calculated best fit normal distribution of maximum dimensions. 

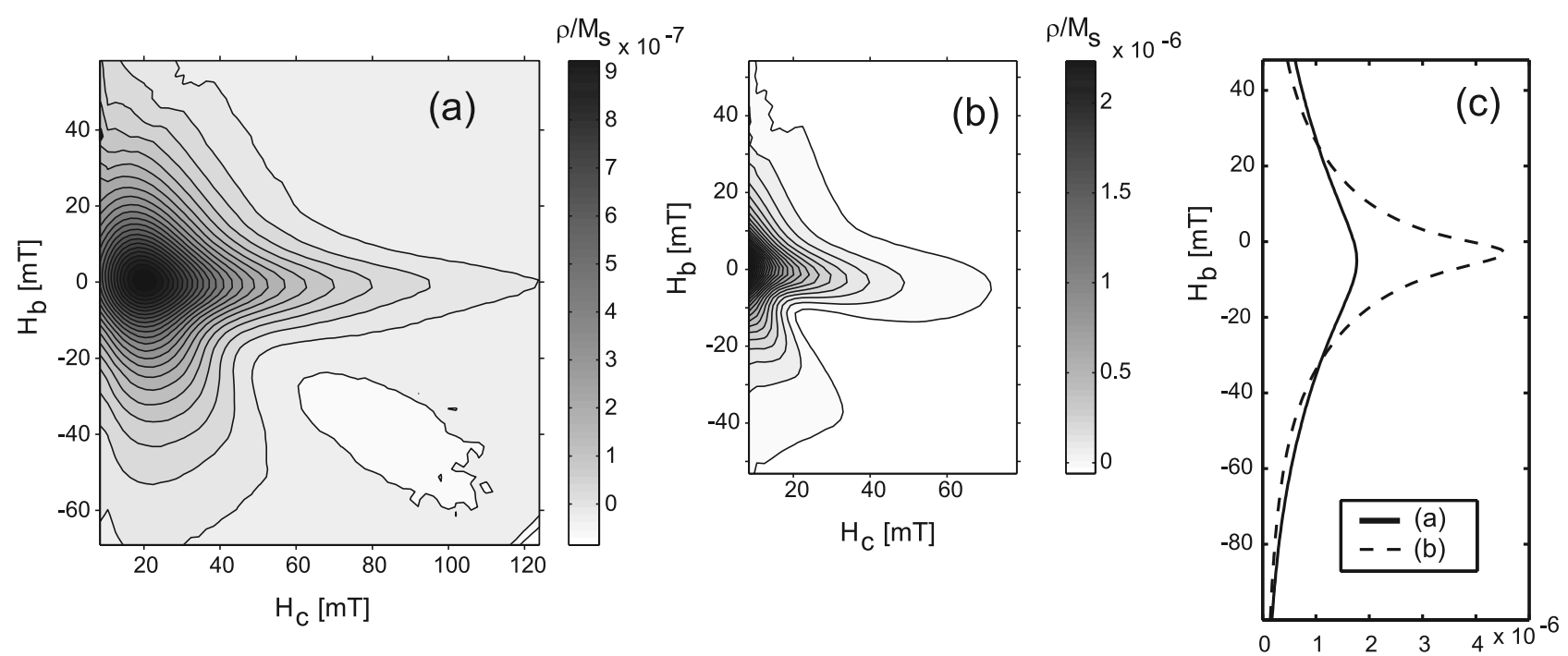

Figure 5. FORC diagrams for (a) the intergrown material and (b) the homogenized material. (c) "Reversible ridge" profiles obtained by extended FORC analysis.

coercivity spectrum appears along the horizontal axis while the distribution of interaction fields causes the spread in the vertical direction. The importance of interactions is conveniently assessed by means of a vertical cross section passing through the coercivity maximum, from which the full width at half maximum (FWHM) is obtained. In our case, $\mathrm{FWHM}=30 \mathrm{mT}$ at $H_{c}=20 \mathrm{mT}$. It is instructive to compare the FORC diagram for the intergrown sample to that obtained by Pike and Fernandez [1999] for Cobalt nanodots. The nanodots have vortex remanence states and are sufficiently isolated to not influence each other by magnetostatic interactions. This results in a highly diagnostic FORC diagram showing the so-called "butterfly structure" that reflects vortex nucleation and annihilation within discrete particles. The absence of such a feature in the FORC diagram for our intergrown sample shows that vortex states have not nucleated within individual particles. The nucleation of a vortex within a particle costs exchange energy, whereas the formation of a supervortex (as found by Harrison et al. [2002]) does not because the center of the vortex is located in the nonmagnetic spacer between the ferromagnetic particles.

[9] The VFTB hysteresis and FORC measurements were repeated using the sample that had been homogenized as described above. The hysteresis loop, IRM acquisition and backfield curves are given in Figures $3 \mathrm{~d}, 3 \mathrm{e}$, and $3 \mathrm{f} . M_{r s} / M_{s}$ has been halved (to 0.11), while $H_{c r} / H_{c}$ and TED have increased to 2.73 and 0.28 , respectively. All of these changes suggest a move toward MD behavior, although $M_{r s} / M_{s}$ and $H_{c r} / H_{c}$ fall short of the threshold values for pure magnetite [Dunlop, 2002]. The FORC diagram (Figure 5b) shows the effect of removing the intergrowths. The interaction profile has dramatically shrunk toward the origin. It is now more difficult to specify the coercivity value through which to draw a profile yielding FWHM because the FORC distribution does not show an isolated maximum any more but instead increases monotonically toward the left margin of the diagram. At $6 \mathrm{mT}$, the lowest significant $H_{c}$ value extracted by the FORC software, we obtain FWHM $=14 \mathrm{mT}$, less than half of that for the intergrown material. Importantly, the FORC contours do not run parallel to the vertical axis, which would be the case for a stress-free multidomain sample [Pike et al., 2001]. In view of the rapid quenching involved, it is quite likely that our FORC diagram reflects the presence of stress in the homogenized titanomagnetite lattice, similar to that reported by Pike et al. [2001] in their comparison of data from magnetite samples before and after annealing (see Pike et al.'s Figure 6). In particular, Figure 6c of Pike et al. [2001] is qualitatively similar to our Figure 5b, although the absolute values on the ordinate are smaller in our case. This difference primarily reflects the fact that our homogenized sample has a lower material specific saturation magnetization than magnetite.

[10] A further point that emerges from an extended version of FORC analysis concerns what Pike [2003] refers to as the "reversible ridge", a concept he introduces to deal with any reversible magnetization present (which "routine" FORC analysis always reduces to zero). The new procedure provides a profile of the interaction fields along the $H_{\mathrm{c}}=0$ axis. Although such components are of little significance when assessing paleomagnetic stability, Pike's procedure is important for a full understanding (and correct normalization) of FORC distributions. The corresponding profiles for our two samples (i.e., before and after homogenization) are illustrated in Figure 5c. It is found that the peak values for both samples are roughly 30 times larger than the peak values in Figures $5 \mathrm{a}$ and $5 \mathrm{~b}$, indicating that reversible magnetization is certainly present and becomes about 3 times larger after homogenization. This probably reflects the relative ease with which domain walls are able to move reversibly in the large homogenized grains.

\section{Discussion}

[11] The hysteresis data are consistent with expectations. In grains larger than several microns (i.e., the laboratoryhomogenized material), domain walls form and the two ratios involved in the Day et al. [1977] plot $\left(M_{r s} / M_{s}\right.$ and 
(a)

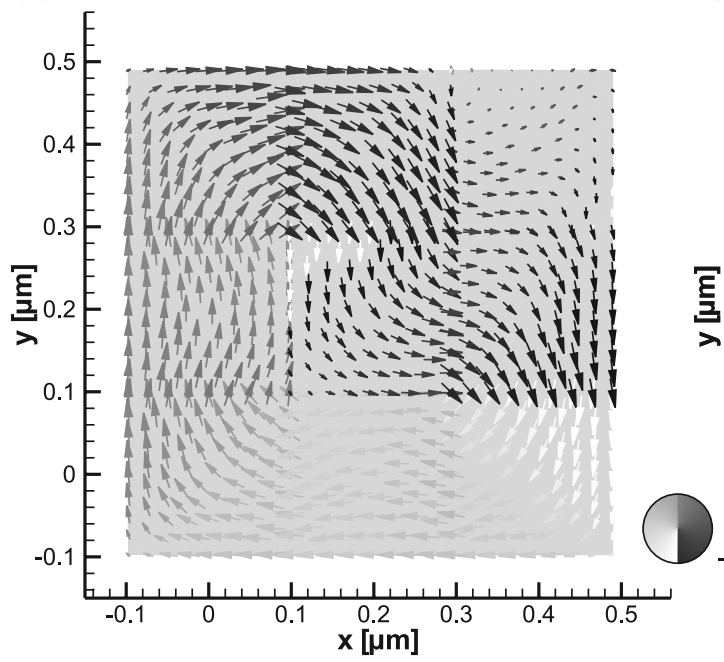

(b)

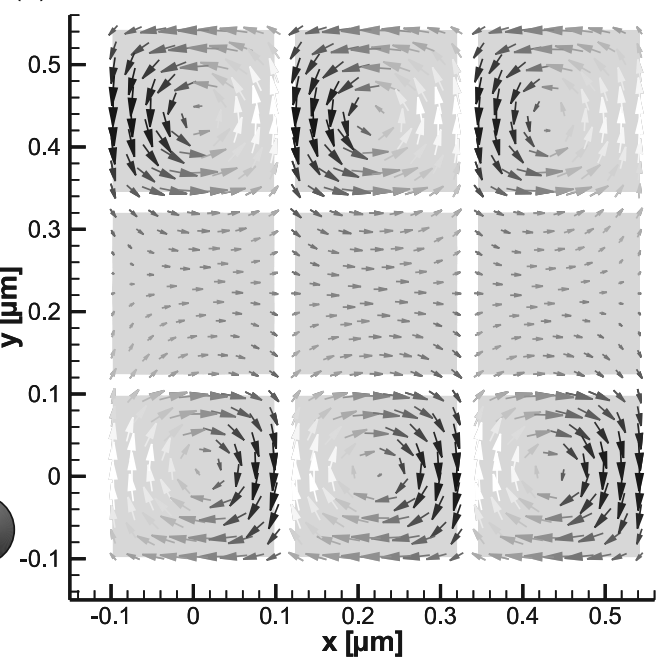

Figure 6. Magnetization structure of two arrays of $3 \times 3$ cubic magnetite particles as it would appear in electron holography. The edge length of the magnetite blocks is in both cases $195 \mathrm{~nm}$ with an interparticle spacing of (a) $1 \mathrm{~nm}$ and (b) $27 \mathrm{~nm}$. Arrow size is proportional to the absolute value of magnetic induction $\mathbf{B}$, the shade gives the orientation of $\mathbf{B}$ as shown by the color wheel.

$H_{c r} / H_{c}$ ) move toward the MD field. On the other hand, the natural starting material consists of grains that are partitioned on a submicroscopic scale into two mineral phases. These take the form of roughly rectangular magnetite blocks separated by thin ulvöspinel walls. (Since the Curie point of ulvöspinel is well below room temperature, it can be regarded as nonmagnetic for the present purposes.) Such a structure behaves as an array of SD and/or PSD particles, but with the important proviso that magnetostatic interactions play a vital role. This is observed both in the vertical spread in the FORC diagram and in the observed $M_{r s} / M_{s}$ ratio for which our VFTB data yield a value of 0.22 , in close agreement to the value of $\sim 0.2$ obtained by Harrison et al. [2002] from their electron holography measurements.

[12] What maximum interaction field might arise in an intergrown grain like that shown in Figure 1? For a simple estimate, consider the field at a distance of $2 r$ (along the $z$ axis) from the center of a spherical SD magnetite region (radius, $r$ ) uniformly magnetized along the $z$ direction. We take $2 r$ in order to be at the center of an identical nearestneighbor particle that is just touching. The field is given by $F=\mu_{0} 2 M / 4 \pi(2 r)^{3}$, where $\mu_{0}$ is the permeability of free space and $M$ is the magnetic moment of the particle. This latter quantity is given by the product $J v$, where $J=480 \mathrm{kA} / \mathrm{m}$ (the spontaneous magnetization of magnetite) and $v$ is the particle's volume. Substitution leads to a field of $\sim 50 \mathrm{mT}$. Furthermore, this value is independent of $r$, as long as the particle remains uniformly magnetized. Despite the limitations of this estimate, it is interesting to note that it agrees remarkably well with the maximum vertical spread observed in the FORC data (FWHM $=30 \mathrm{mT}$ at $H_{c}=20 \mathrm{mT}$, Figure 5).

[13] As a further check, we carried out several fully threedimensional micromagnetic calculations on relevant grain configurations. The calculations were based on a finite element grid consisting of irregular four-node tetrahedral elements with an average node spacing of $25 \mathrm{~nm}$. We used a combination of minimum energy conjugate gradient $(\mathrm{CG})$ algorithm and dynamic algorithm solving the LandauLifshitz-Gilbert (LLG) equation [Muxworthy and Williams, 2005]. In order to simulate the exsolved material we constructed an array of 3 by 3 cubic particles of magnetite with an edge length of $195 \mathrm{~nm}$ (median grain size of exsolved blocks). The particles have cubic anisotropy with the easy axes oriented along the [111] directions, where the grains lie in the (001) plane. One calculation was conducted for virtually touching particles (intergrain spacing $1 \mathrm{~nm}$ ), the other for a spacing of $27 \mathrm{~nm}$, the microscopically determined median value for the exsolved titanomagnetite. In both cases, the external magnetic field was set to zero. The magnetization structure as it would appear in electron holography imaging is shown in Figure 6 and was determined for all (001) surface nodes by integrating the magnetic induction along the $z$ axis.

[14] In the case of the wider spacing, all nine particles are in the vortex state, as would be expected for noninteracting particles with the same grain size. Particles in the upper and lower row have their vortex axes oriented along the $z$ axis whereas those of particles of the middle row are oriented along the $x$ axis. The situation is different for closely spaced particles of the second model where the narrow gaps between particles make the formation of a supervortex state, comparable to the ones observed by Harrison et al. [2002], energetically more favorable. As expected, the core of this supervortex is located in the gap between magnetite blocks at the boundary between the outer particles and the center particle in the lower left corner of the array. The particle in the upper right corner does not fully participate in this magnetization superstate.

[15] The different magnetization structures are also reflected in the interaction field exerted on a given particle by the adjacent particles (Figure 7). The average absolute value of the interaction field $H_{\text {int }}$ acting on the center particle in the case of touching particles is $0.290 J_{\mathrm{S}}(J$ being the saturation magnetization) which corresponds to $\sim 175 \mathrm{mT}$. For the $27 \mathrm{~nm}$ spacing model, the average interaction 
(a)

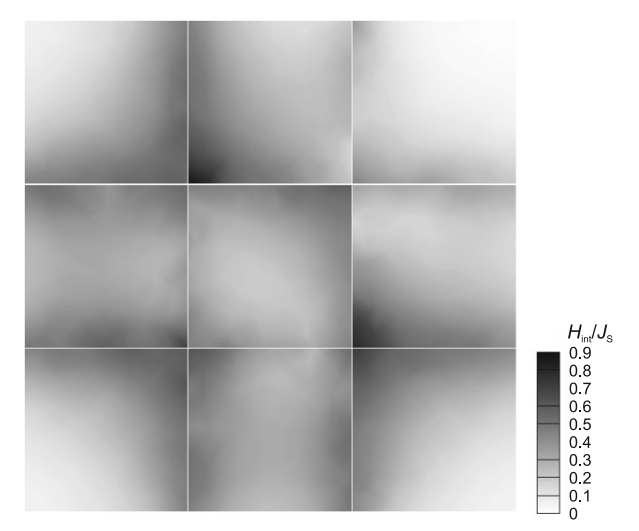

(b)

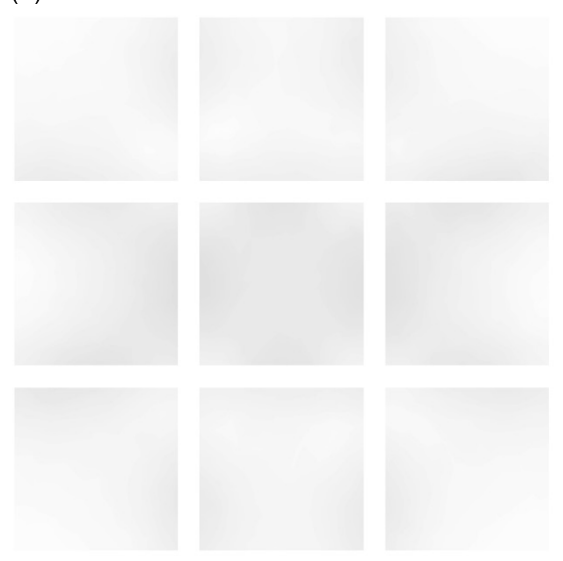

(c)

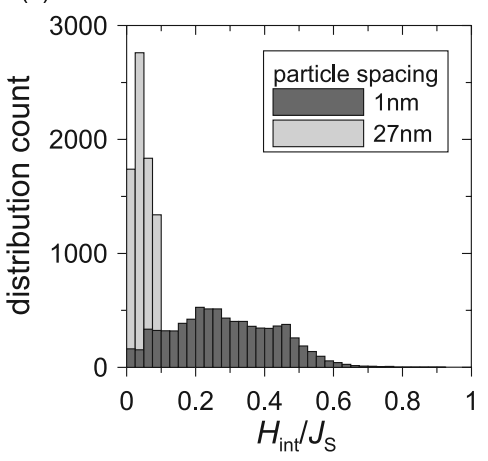

Figure 7. $(\mathrm{a}, \mathrm{b})$ Map of the absolute value of the interaction field exerted on a given element of a given particle by the adjacent particles: particle spacing $1 \mathrm{~nm}$ (Figure $7 \mathrm{a}$ ) and $27 \mathrm{~nm}$ (Figure $7 \mathrm{~b}$ ). In the case of wider spacing, the average interaction field is reduced both by the larger distance between the particles as well as by the vortex state adopted by the individual particles. (c) Histograms of interaction field distribution for the arrays of particles with 1-nm spacing and 27-nm spacing, respectively.

field for the center particle is less than a third of this value with $H_{\text {int }}=0.082 J_{\mathrm{S}}(\sim 50 \mathrm{mT})$. If the whole array of particles were uniformly magnetized, the difference would be much less pronounced, namely, $H_{\text {int }}=0.230 J_{\mathrm{S}}$ for $1 \mathrm{~nm}$ spacing and $H_{\mathrm{int}}=0.156 J_{\mathrm{S}}$ for $27 \mathrm{~nm}$ spacing. This signifies that the difference in magnetostatic interaction is not only due to the $1 / r^{3}$ dependence of the magnetostatic field but also due to the fact that the magnetization structure of individual particles changes from a coherent state to the vortex state with increasing particle separation. The latter strongly reduces the magnetic stray field. Therefore grain separation does control magnetostatic interactions in a more decisive way in the present case than it would for an array of smaller particles in the stable single-domain grain size range.

[16] Our micromagnetic models suggest that only those grains within our sample which are smaller than the median grain size, or whose intergrain separation is significantly less than the average, will have supervortex states. A closer examination of the grain dispersion within our sample (Figure 1) shows that large isolated grains are relatively rare, with most grains being in clusters of near-touching grains. Thus most grains should be in supervortex domain states, dominated by intergrain magnetostatic interactions, with relatively few isolated grains in a SD or vortex state which is compatible with the FORC results.

\section{Conclusions}

[17] We have investigated the effect of magnetostatic interactions in a naturally occurring ultrafine intergrown material consisting of arrays of quasi-rectangular magnetite blocks separated by ulvöspinel lamellae. Transmission electron microscopy indicates that the magnetite blocks have a Gaussian size distribution with a median value for their maximum dimension of $195 \mathrm{~nm}$ and an exponential distribution for the gaps between them with a median value of $27 \mathrm{~nm}$. Hysteresis parameters are $0.22,1.98$ and 0.18 for $M_{r s} / M_{s}, H_{c r} / H_{c}$, and TED, respectively. The FORC diagram provides a direct measure of the interaction fields, giving a value for the full width at half maximum (FWHM) of $30 \mathrm{mT}$ (at $H_{c}=20 \mathrm{mT}$ ). After elimination of the intergrowth structure, by homogenizing the powder in a sealed vial, we obtain values of $0.11,2.73$ and 0.28 for $M_{r s} / M_{s}, H_{c r} / H_{c}$, and TED, respectively. Furthermore, the contours on the FORC diagram shrink down toward the origin, yielding FWHM = $14 \mathrm{mT}$ (at $H_{c}=6 \mathrm{mT}$ ). All these characteristics reveal the strong influence of particle-to-particle magnetostatic interactions between the magnetite blocks in the naturally occurring starting material and demonstrate the change from $\mathrm{SD} / \mathrm{PSD}$ to MD behavior due to the fundamental structural change brought about by the experimental homogenization. The importance of magnetostatic interactions in the original intergrowth is also evident from micromagnetic calculations of appropriate grain configurations.

[18] Acknowledgments. We are grateful to Cor Langereis and Tom Mullender for much help in carrying out the FORC measurements at the "Fort." The paper was improved by constructive reviews by Adrian Muxworthy and Joshua Feinberg. Financial support to MEE was provided by the Natural Sciences and Engineering Council of Canada, and by a UK Natural Environment Research Council grant (NE/C510159/1) to W.W. and D.K.

\section{References}

Basta, E. Z. (1960), Natural and synthetic titanomagnetites, Neues Jahrb. Mineral. Abh., 94, 1017-1048.

Day, R., M. Fuller, and V. A. Schmidt (1977), Hysteresis properties of titanomagnetites: Grain-size and compositional dependence, Phys. Earth Planet. Inter, 13, 260-267.

Dunlop, D. J. (2002), Theory and application of the Day plot $\left(M_{\mathrm{rs}} / M_{\mathrm{S}}\right.$ versus $\left.H_{\mathrm{cr}} / H_{\mathrm{c}}\right): 1$. Theoretical curves and tests using titanomagnetite data, J. Geophys. Res., 107(B3), 2056, doi:10.1029/2001JB000486.

Evans, M. E., and M. L. Wayman (1974), An investigation of the role of ultra-fine titanomagnetite intergrowths in palaeomagnetism, Geophys. J. R. Astron. Soc., 36, 1-10.

Fabian, K. (2003), Some additional parameters to estimate domain state from isothermal magnetization measurements, Earth Planet. Sci. Lett., 213, 337-345.

Fabian, K., A. Kirchner, W. Williams, F. Heider, T. Leibl, and A. Huber (1996), Three-dimensional micromagnetic calculations for magnetite using FFT, Geophys. J. Int., 124, 89-104.

Feinberg, J. M., G. R. Scott, P. R. Renne, and H. R. Wenk (2005), Exsolved magnetite inclusions in silicates: Features determining their remanence behavior, Geology, 33, 513-516. 
Harrison, R. J., R. E. Dunin-Borkowski, and A. Putnis (2002), Direct imaging of nanoscale magnetic interactions in minerals, Proc. Natl. Acad. Sci. U.S. A., 99, 16,556-16,561.

Moskowitz, B. M. (1981), Methods for estimating Curie temperatures of titanomaghemites from experimental $\mathrm{J}_{\mathrm{s}} \mathrm{T}$ data, Earth Planet. Sci. Lett., $53,84-88$.

Muxworthy, A., and W. Williams (2005), Magnetostatic interaction fields in first-order-reversal-curve diagrams, J. Appl. Phys., 97, 063905, doi:10.1063/1.1861518.

Muxworthy, A., D. Heslop, and W. Williams (2004), Influence of magnetostatic interactions on first-order-reversal-curve (FORC) diagrams: A micromagnetic approach, Geophys. J. Int., 158, 888-897.

Pike, C. R. (2003), First-order reversal-curve diagrams and reversible magnetization, Phys. Rev. B, 68, 104424.

Pike, C. R., and A. Fernandez (1999), An investigation of magnetic reversal in submicron-scale Co dots using first-order reversal-curve diagrams, J. Appl. Phys., 85, 6668-6675.

Pike, C. R., A. P. Roberts, and K. L. Verosub (1999), Characterizing interactions in fine magnetic particle systems using first order reversal curves, J. Appl. Phys., 85, 6660-6667.
Pike, C. R., A. P. Roberts, M. J. Dekkers, and K. L. Verosub (2001), An investigation of multi-domain hysteresis mechanisms using FORC diagrams, Phys. Earth Planet. Inter., 126, 11-25.

Preisach, F. (1935), Über die magnetische Nachwirkung, Z. Phys., 94, $277-$ 302.

Roberts, A. P., C. R. Pike, and K. L. Verosub (2000), First-order reversal curve diagrams: A new tool for characterizing the magnetic properties of natural samples, J. Geophys. Res., 105, 28,461-28,475.

M. E. Evans, Department of Physics, University of Alberta, Edmonton, AB, Canada T6G 2J1.

D. Krása and W. Williams, School of GeoSciences, University of Edinburgh, King's Buildings, Edinburgh EH9 3JW, UK.

M. Winklhofer, Department of Earth and Environmental Sciences, Ludwig-Maximilians-Universität, Theresienstr. 41, D-80333 Munich, Germany. 